\title{
Validation of the beat to beat measurement of blood velocity in the human ascending aorta by a new high temporal resolution Doppler ultrasound spectral analyser
}

\author{
A J S Coats, C Murphy, J Conway, P Sleight
}

\begin{abstract}
Objective-To develop and validate a high temporal resolution spectral analysis system for Doppler measurements of blood velocity in the ascending aorta.

Design-An observational laboratory and clinical study comparing Doppler velocity-based measurements with fluid collection, electromagnetic flow catheters and probes, and thermodilution.

Setting-Tertiary referral cardiology unit and cardiac catheter laboratory.

Patients-Patients undergoing routine cardiac catheterisation for ischaemic heart disease, cardiac failure, and primary pulmonary hypertension.

Results-There was good agreement between Doppler-derived and electromagnetic cuff or catheter measurements of velocity in an experimental flow rig (SD of differences $4 \cdot 75 \%$ for velocity integral) and in the patients (SD of differences $4 \%$ for velocity integral). There was also reasonably good agreement between simultaneous Dopplerderived and thermodilution-estimated cardiac output measurements in patients undergoing cardiac catheterisation (SD of differences $12 \cdot 6 \%$ ).

Conclusions-This new method of high temporal resolution spectral analysis improves the resolution of rapidly changing blood velocities and may improve the ability to describe blood velocity patterns in the ascending aorta.
\end{abstract}

(Br Heart J 1992;68:223-9)

Department of Cardiovascular Medicine, John Radcliffe Hospital, Oxford

A J S Coats

C Murphy

J Conway

P Sleight

Correspondence to Dr J S Coats, Department of Cardiac Medicine, National Heart and Lung Institute, London SW3 $6 \mathrm{LY}$

Accepted for publication 3 February 1992
Doppler ultrasound has been used extensively in the assessment of normal and abnormal flow patterns in humans. ${ }^{1}$ Although for many routine clinical uses continuous wave Doppler machines are often adequate, to obtain quantitative information on the pattern of blood velocities in the heart or ascending aorta accurate localisation of the sampled velocity is important; hence the distance resolution of pulsed wave Doppler ultrasound is critical. In addition, the use of blood velocity estimates to calculate volume flow, blood acceleration, or the pattern of the aortic velocity wave requires good temporal as well as spatial resolution. Conventional combined Doppler echocardiographic machines either share time between the Doppler and imaging modes, use the same transducer with compromised characteristics for both imaging and Doppler ultrasound, or have low temporal resolution spectral analysers (with sampling every 5-20 ms).

Some of the simpler methods of spectral analysis used in early studies are subject to systematic errors. The zero-crossing detector, for example, can miss high frequency signals when they are superimposed on low frequency signals, because the higher frequency sound wave may not cause the pressure signal to cross zero. The most accurate method of spectral analysis is complete resolution of the different spectral components by fast Fourier transformation (FFT). FFT is now available in realtime computer processing because of the development of powerful minicomputers.

Temporal resolution in spectral analysis can be improved by more frequent FFT analyses, but each time bin must be large enough to include one full cycle of the lowest frequency to be resolved. To detect frequencies down to $100-200 \mathrm{~Hz}$, therefore, the time bin can be reduced to no shorter than $5 \mathrm{~ms}$. Even this resolution may be inadequate to resolve changing blood velocities during the acceleration phase of cardiac ejection because the blood velocity varies from zero to $1-1.5 \mathrm{~ms}$ in $50 \mathrm{~ms}$ or less. We have developed, therefore, a system with shifting analyses to improve time resolution. $^{2}$

Physiologically it may be important to resolve blood velocities to this greater degree because turbulence and rapidly changing velocities in blood flow can be better detected and quantified. It has been proposed that turbulence may be important in the development of atherosclerotic plaques ${ }^{3}$ as well as contributing to the impedance to ejection of blood by the ventricle. ${ }^{4}$ Much has been written on the importance of aortic blood acceleration in the assessment of left ventricular function, ${ }^{5-}$ ${ }^{7}$ yet little attention is directed to the problems of accurate measurement of aortic blood acceleration. Acceleration is variously defined as the mean acceleration over the time from velocity onset to peak velocity, ${ }^{8}$ the mean 

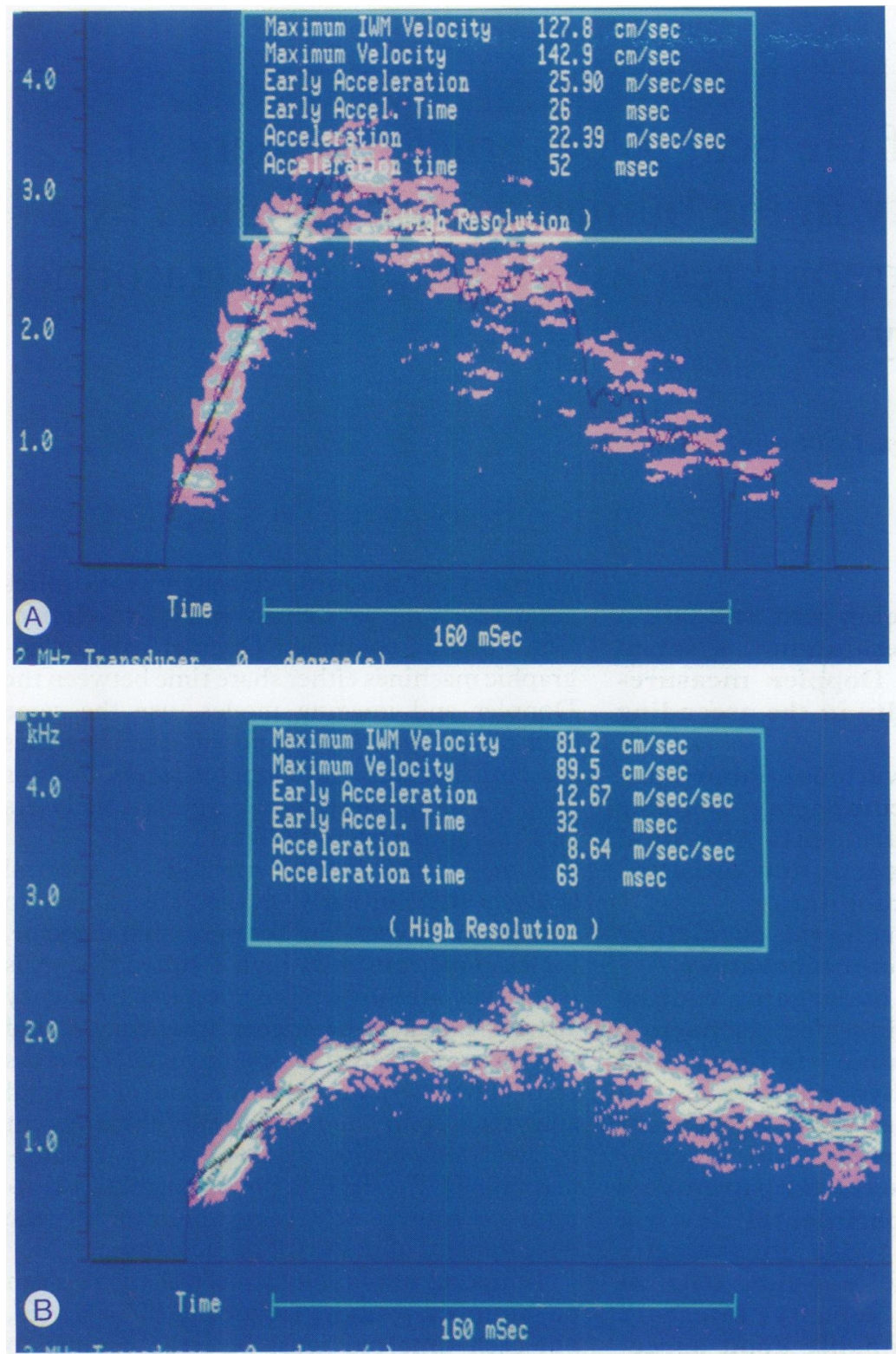

Figure 1 A high resolution Doppler spectral display blood velocity in the ascending aorta in two healthy individuals at rest. $(A)$ a 17 year old woman and $(B)$ a 47 year old man. Single beats were analysed off-line by the high resolution mode. The $Y$ axes are marked in $\mathrm{kHz}$ representing the Doppler frequency shifts, which are directly.

proportional to the velocity of blood in the direction of the ultrasound. Time is marked on the $X$ axis. The table at the top is a summary of the analysis of various variables derived from the single beat shown. IWM, intensity weighted mean.

acceleration over the initial part of the acceleration phase when acceleration seems to be close to constant, ${ }^{8-10}$ or the "peak instantaneous acceleration". ${ }^{6}$ All three methods are subject to considerable errors because of the low temporal resolution of previous spectral analysers. Figure 1 shows that the upstroke of aortic blood velocity is often curvilinear with no clear point of peak velocity. Acceleration is therefore subject to considerable errors when there is noise at the point of peak velocity. No information can be obtained on the rate of change of velocity before the high pass filter (100$400 \mathrm{~Hz}$ ), and hence acceleration based on the first half of the acceleration phase may be based on only $20-25 \mathrm{~ms}$ of data and may therefore depend on only two or three separate spectral analyses, so that noise in any one time bin would cause considerable uncertainty in the overall assessment of acceleration. Without high temporal resolution spectral analysis measurements of "instantaneous peak acceleration" are even more liable to error and artefact in that instantaneous peak acceleration effectively means the greatest change in velocity between any two time bins and hence is extremely subject to noise in either time bin, especially the first where no velocities below the high pass filter can be detected. It is also fallacious to refer to peak acceleration as being "instantaneous" when temporal resolution is as low as $5-20 \mathrm{~ms}$ and changes in aortic blood velocity can occur over less than $5 \mathrm{~ms}$.

\section{Methods}

DOPPLER EQUIPMENT

These studies were performed with standalone non-imaging Alfred or Pedof Doppler ultrasound transducers (Vingmed, Norway) with a $2 \mathrm{MHz}$ probe, and a spectral analysis program specifically developed in the hardware environment of a Texas Instruments personal computer (Texas Instruments, Texas, USA). The analyser design and specifications have been described. ${ }^{211}$ We have improved the temporal resolution of the spectral analyser by storing the original reflected ultrasound signal directly to computer memory, while simultaneously displaying the on-line spectrally analysed signal to aid signal acquisition. ${ }^{2}$ At the end of signal acquisition the original ultrasound information is re-analysed in $5 \mathrm{~ms}$ bins, followed by repeated re-analysis with the bin being shifted $0 \cdot 1 \mathrm{~ms}$ each time. This allows a full $5 \mathrm{~ms}$ of signal to be analysed each time, but also allows rapidly changing frequencies to be resolved because the time bins shift only $0.1 \mathrm{~ms}$ at a time. The resulting temporal resolution lies between 0.1 and $5 \mathrm{~ms}$, and is approximately $1 \mathrm{~ms}$.

VALIDATION AGAINST PURE SOUND FREQUENCIES The spectral analyser received pure sound signals from a high frequency sweep generator model 144 (Wavetek, USA) with static and changing frequencies. We compared the frequency resolution and ability to follow changing frequencies of a commercial analyser (Doptek, Chichester, UK) on fast and slow sweep settings, the Texas instruments analyser with normal on-line spectral analysis resolution, and the Texas instruments analyser with immediate off-line high temporal resolution analysis. We studied the ability to resolve frequencies varying between 0 and $1 \mathrm{kHz}$ by using square wave pulses between 0 and $1 \mathrm{kHz}$, sweeps between the two frequencies, and continuous triangular waves oscillations between the two frequencies.

\section{VALIDATION AGAINST A FLOW RIG}

A flow rig was designed (fig 2) to mimic the ascending aorta of adult man. It used a pulsatile pump to eject a suspension of Sephadex. At a range of pump stroke distances of $38-54 \mathrm{~cm}$ and power settings of 6 to $15 \mathrm{~V}$. Five pump beats were measured by three methods: Dop- 


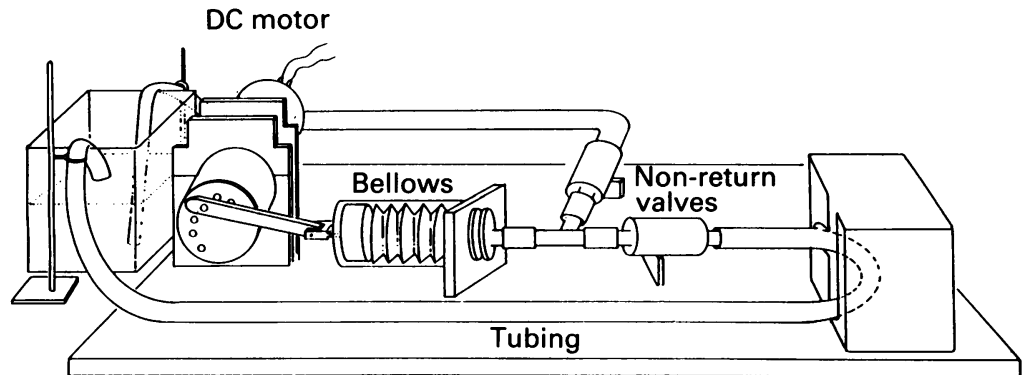

Figure 2 Pulsatile flow rig used to test Doppler velocity and flow measurements against electromagnetic cuff measurements and volume flow collection.

pler ultrasonic measurement of fluid velocity, electromagnetic cuff (Medical Flow Meter System with SEM 275, Feltham, Middlesex) measurement of fluid velocity, and volume flow by fluid collection. The Doppler ultrasound was focused so that the pulse volume recorded velocities occurring as the suspension travelled through the electromagnetic cuff, the internal diameter of which was known. Both Doppler and electromagnetic velocity integrals were converted to volume estimates by multiplication with the internal cross sectional area of the electromagnetic cuff. For the comparison of Doppler and electromagnetic measurements both peak flow and stroke volume were compared, and both measurements of volume flow were compared with absolute flow collected over five beats.

\section{VALIDATION OF VELOCITY VARIABLES IN PATIENTS}

In six men with coronary artery disease (aged 52-73) diagnostic cardiac catheterisation was followed by insertion of an electromagnetic flow catheter to the ascending aorta and simultaneous readings were obtained by the electromagnetic catheter (Mills catheter with SEM 275 analyser, Feltham, Middlesex) and pulsed wave Doppler ultrasound of blood velocity in the ascending aorta from the suprasternal approach. Unselected cardiac beats $(\geq 100)$ were recorded for each subject at rest and during various procedures including: programmed electrical stimulation producing extrasystoles and post-extrasystolic accentuated complexes; head up and head down tilting; and inotropic drug stimulation (intravenous milrinone). The interventions with vasoactive drugs were part of routine management or part of separate research protocols for which ethical approval and patient consent had been obtained.

\section{VALIDATION OF VOLUME FLOW CALCULATIONS} IN PATIENTS

In 11 patients undergoing right heart catheterisation 64 simultaneous thermodilution (Gould haemodynamic profile computer) and Dopplerderived measurements of cardiac output were obtained at rest and after various procedures such as head up and head down tilting, inotropic drug stimulation (intravenous milrinone), and vasodilator drug administration (intravenous prostacyclin or oral nifedipine). The conditions for which the patients were undergoing cardiac catheterisation were ischaemic heart disease (9), congestive heart failure (5), and primary pulmonary hypertension (2). All patients gave informed consent for these studies, for which ethical committee approval had been given. The age range of patients was from 38 to 73 years and all were in stable sinus rhythm.

The aortic cross sectional area was estimated on a separate occasion within 24 hours of the Doppler recording by means of the leading edge to leading edge convention with cross sectionally directed $M$ mode echocardiographic imaging at the level immediately above the sinuses of Valsalva in mid-systole. ${ }^{12-14}$

\section{STATISTICAL METHODS}

Comparisons between methods were examined by the method of Bland and Altman. ${ }^{15}$ Systematic differences between methods were tested for by Student's $t$ test (two tailed), a $p$ value of $\leqslant 0.05$ was regarded as statistically significant.

\section{Results}

VALIDATION AGAINST PURE SOUND FREQUENCIES The spectral analyser received pure sound signals with static and changing frequencies. Rapidly changing frequencies were presented to four different modes of spectral analyser: the commercial Doptek analyser on slow and fast sweep settings (spectral analysis time bins of approximately 10 and $5 \mathrm{~ms}$ respectively) and the Texas instruments analyser on normal temporal resolution and on immediate off-line high temporal resolution mode. Table 1 shows that as the rate of change of frequency increases the lower resolution modes cease to be able to distinguish the rapidly changing frequencies and the spectral display becomes a blur spreading from 0 to $1 \mathrm{kHz}$. This is shown in fig $3 \mathrm{~A}$ in which the signal was analysed by a conventional resolution analyser and is perceived as a wide-band blur of frequencies. The high temporal resolution mode can, however, still determine that the signal is a rapidly changing frequency and the shape of rapidly changing frequency slope is apparent even when it varies from 0 to $1 \mathrm{kHz}$ at a rate of at least 100 times a second (fig 3B). Table 1 lists the changing frequencies that were resolved adequately by each analysis mode.

Table 1 Ability of four modes of spectral analysis temporally to resolve changing frequencies

\begin{tabular}{llllll}
\hline & \multicolumn{2}{l}{ Doptek analyser } & & \multicolumn{2}{c}{ Texas analyser } \\
\cline { 2 - 3 } Mode & Slow & Fast & & Normal & High Res \\
\hline Duration (ms): & & & & & $1,2,3$ \\
5 & - & - & & $1,2,3$ \\
10 & 1 & $1,2,3$ & $1,2,3$ & $1,2,3$ \\
15 & $1,2,3$ & $1,2,3$ & & $1,2,3$ & $1,2,3$ \\
20 & & &
\end{tabular}

$1=$ adequately resolved a square pulse of $1 \mathrm{kHz}$ that lasted the duration specified under the column duration; $2=$ adequately resolved a sweep between 0 and $1 \mathrm{kHz}$ that lasted the same duration; and $3=$ adequately resolved a triangular wave continually varying between 0 and $1 \mathrm{kHz}$ with each 0 to 1 to $0 \mathrm{kHz}$ cycle lasting the duration specified under the duration column. Where none of the signals was adequately resolved a minus sign is shown. 

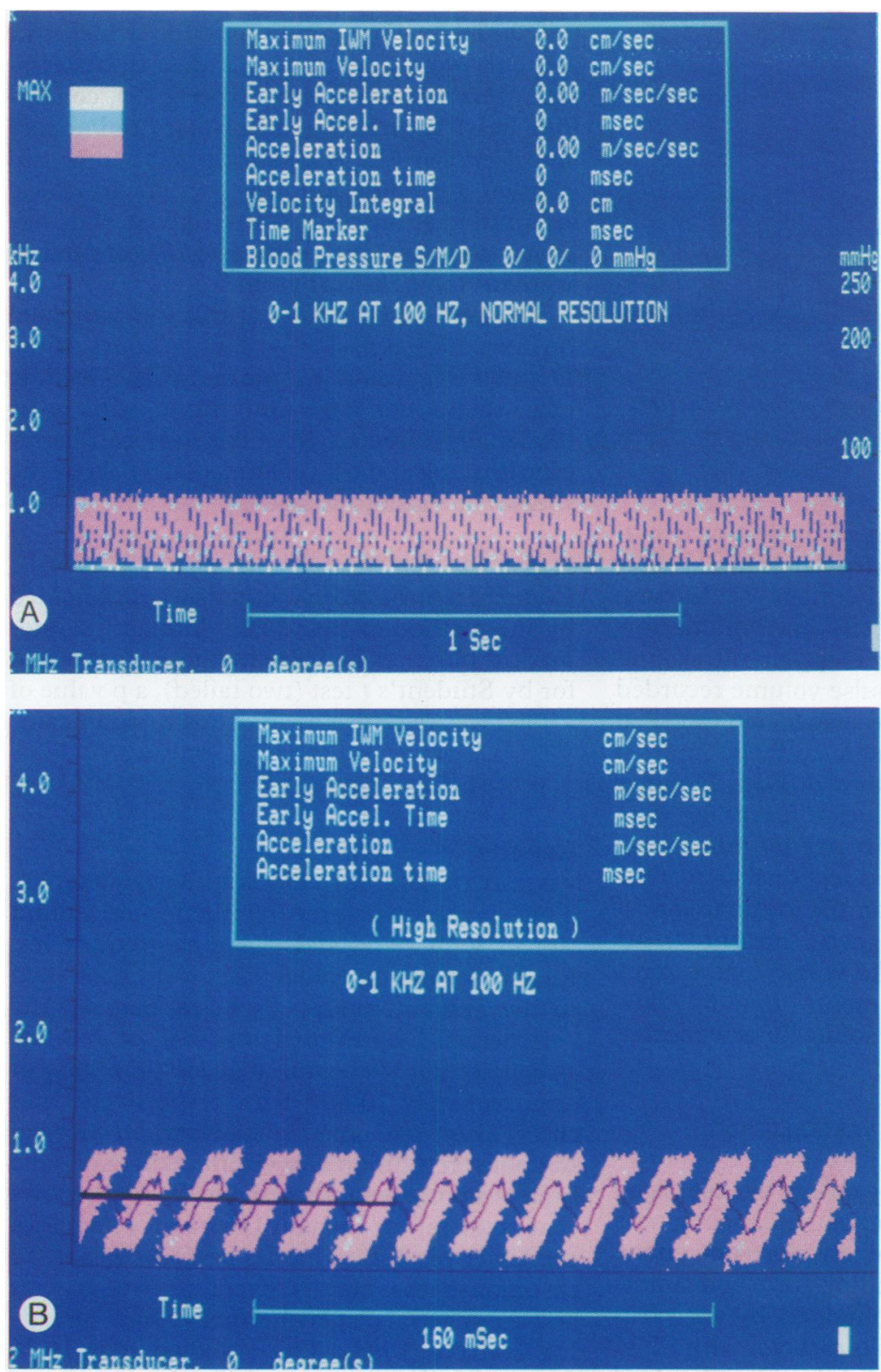

Figure 3 Frequency signals varying from 0 to $1 \mathrm{kHz}$ at 100 times per second (each sweep lasting $10 \mathrm{~ms}$ ) analysed on the Texas Instruments analysis system with ( $A$ normal resolution and $(B)$ high resolution modes. During the normal resolution mode the signal is blurred whereas in high resolution mode the saw-tooth variation in frequency can be resolved.
Table 2 Results of flow rig experiment

\begin{tabular}{|c|c|c|c|c|}
\hline \multirow[b]{2}{*}{ Run } & \multicolumn{2}{|c|}{ Electromagnetic cuff } & \multicolumn{2}{|l|}{ Doppler } \\
\hline & $P F(\mathrm{ml} / \mathrm{s})$ & $S V(m l)$ & $P F(m l / s)$ & $S V(m l)$ \\
\hline $\begin{array}{r}1 \\
2 \\
3 \\
4 \\
5 \\
6 \\
7 \\
8 \\
9 \\
10 \\
11 \\
12 \\
13 \\
14\end{array}$ & $\begin{array}{r}90.4 \\
141.4 \\
186.0 \\
239.6 \\
80.8 \\
115.6 \\
145.6 \\
190.8 \\
104.8 \\
164 \cdot 2 \\
222.2 \\
277.0 \\
205.2 \\
123.6\end{array}$ & $\begin{array}{l}68 \cdot 8 \\
73 \cdot 2 \\
80 \cdot 7 \\
81 \cdot 5 \\
55 \cdot 6 \\
61 \cdot 0 \\
64 \cdot 6 \\
69 \cdot 2 \\
81 \cdot 9 \\
89 \cdot 2 \\
91 \cdot 5 \\
95 \cdot 8 \\
75 \cdot 6 \\
37 \cdot 3\end{array}$ & $\begin{array}{r}101.9 \\
149.9 \\
187.6 \\
223.6 \\
86.0 \\
121.6 \\
148.1 \\
185.7 \\
114.0 \\
163.1 \\
211.3 \\
258.4 \\
191.4 \\
111.2\end{array}$ & $\begin{array}{l}70 \cdot 4 \\
77 \cdot 3 \\
81 \cdot 8 \\
81 \cdot 3 \\
56 \cdot 1 \\
60 \cdot 4 \\
67 \cdot 0 \\
69 \cdot 5 \\
76 \cdot 5 \\
84 \cdot 3 \\
91 \cdot 0 \\
91 \cdot 0 \\
66 \cdot 6 \\
36 \cdot 6\end{array}$ \\
\hline
\end{tabular}

Each value is the mean of five beats per run. Runs $1-4$ were performed with the stroke distance of the piston set at $46 \mathrm{~cm}$ and the power of the pump progressively increased from $6.0 \mathrm{~V}$ to $9 \cdot 0,12 \cdot 0$, and $15.0 \mathrm{~V}$. Runs $5-8$ used the same progressive voltage settings with stroke distance at $38 \mathrm{~cm}$, and for runs 9-12 the stroke distance was set at $54 \mathrm{~cm}$. Run 11 was at $12.0 \mathrm{~V}$ at a stroke distance $54 \mathrm{~cm}$ with constriction of the outflow tubing and run 12 was similar with constriction of the inflow tubing. PF, peak flow; SV, stroke volume.

shown by Doppler and electromagnetic cuff were similar (see single pump beat in fig 4).

VALIDATION OF VELOCITY VARIABLES IN VIVO Figure 5 shows the comparisons between electromagnetic catheter derived and Doppler derived peak velocity, acceleration, and stroke distance for 621 unselected beats from all subjects ( 521 beats for acceleration because in one subject electrical interference on the tape made the Doppler estimate of acceleration impossible) using the Bland and Altman plot method. The mean and standard deviations of the differences between the Doppler and electromagnetic catheter methods were $-\mathbf{0 . 0 5}$ (6.5)\% for peak velocity; $-0.4(8.7) \%$ for stroke distance; and $-1.9(13.7) \%$ for acceleration. Estimates of minute distance were the averages of 10 consecutive cardiac beats. The mean \pm (SD) of differences between the two methods was $-0.67(3.6) \%$ (fig 6 ).

Table 5 shows the correlation coefficients between variables measured by Doppler and electromagnetic methods for individual subjects.

VALIDATION OF VOLUME FLOW CALCULATIONS IN VIVO

The results of all simultaneous measurements between Doppler derived cardiac output and

Table 2 compares measurements of stroke volume and peak flow by the electromagnetic cuff and the Texas Instruments Doppler system in the flow rig. The methods were compared according to the recommendations of Bland and Altman. ${ }^{15}$

They did not give significantly different results (electromagnetic first): mean (SD) peak velocity $163.4(59 \cdot 1) \mathrm{ml} / \mathrm{s}$ compared with $161 \cdot 0$ $(51.0) \mathrm{ml} / \mathrm{s}$ (NS); mean (SD) stroke volume $73 \cdot 3(15 \cdot 6)$ compared with $72 \cdot 1$ (14.7) NS.

Table 3 shows the comparison between either method and the five beat pump volume. Table 4 shows the comparison by Bland and Altman's method. Other conditions mimicking clinical conditions were investigated including outflow (run 13) and inflow constriction (run 14) (table 3 ). The shapes of the velocity profiles

Table 3 Volume flow estimates in the rig by electromagnetic cuff, Doppler, and fluid collection

\begin{tabular}{llll}
\hline Run & $\begin{array}{l}\text { E/M cuff } \\
(\text { l/min })\end{array}$ & $\begin{array}{l}\text { Doppler } \\
(l / \text { min })\end{array}$ & $\begin{array}{l}\text { Fluid collection } \\
(\text { l/min })\end{array}$ \\
\hline 1 & 1.21 & 1.24 & 1.15 \\
2 & 2.04 & 2.15 & 1.97 \\
3 & 3.07 & 3.11 & 2.92 \\
4 & 3.95 & 3.94 & 3.77 \\
5 & 1.00 & 1.00 & 0.89 \\
6 & 1.70 & 1.68 & 1.53 \\
7 & 2.46 & 2.55 & 2.32 \\
8 & 3.35 & 3.36 & 3.23 \\
9 & 1.41 & 1.32 & 1.21 \\
10 & 2.48 & 2.34 & 2.30 \\
11 & 3.48 & 3.46 & 3.25 \\
12 & 4.56 & 4.33 & 4.34 \\
13 & 2.89 & 2.53 & 2.70 \\
14 & 1.44 & 1.41 & 1.35 \\
\hline
\end{tabular}

See footnote to table 2 for explanation of runs. 
Table 4 Volume flow and flow variables expressed as the mean absolute and percentage differences between methods and the standard deviations of these differences according to the recommendations of Bland and Altman (1986) ${ }^{15}$

\begin{tabular}{|c|c|c|}
\hline & Mean difference & $S D$ difference \\
\hline$D-E M$ & \multicolumn{2}{|l|}{ Flow rate $(l / \min )$} \\
\hline $\begin{array}{l}\text { Absolute } \\
\text { Percentage }\end{array}$ & $\begin{array}{l}-0.041 \\
-1.43\end{array}$ & $\begin{array}{l}0.124 \\
4.75\end{array}$ \\
\hline $\begin{array}{l}\text { D - Coll: } \\
\text { Absolute } \\
\text { Percentage }\end{array}$ & $\begin{array}{l}0 \cdot 106 \\
5 \cdot 38\end{array}$ & $\begin{array}{l}0.101 \\
4.69\end{array}$ \\
\hline $\begin{array}{c}\text { EM - Coll: } \\
\text { Absolute } \\
\text { Percentage }\end{array}$ & $\begin{array}{l}0 \cdot 147 \\
6 \cdot 81\end{array}$ & $\begin{array}{l}0.054 \\
3.24\end{array}$ \\
\hline & \multicolumn{2}{|l|}{ Peak flow $(\mathrm{ml} / \mathrm{s})$} \\
\hline $\begin{array}{l}\text { - EM: } \\
\text { Absolute } \\
\text { Percentage }\end{array}$ & $\begin{array}{l}-2.39 \\
-1.60\end{array}$ & $\begin{array}{r}10 \cdot 29 \\
6 \cdot 79\end{array}$ \\
\hline & \multicolumn{2}{|l|}{ Stroke volume ( $\mathrm{ml}$ ) } \\
\hline $\begin{array}{l}\text { Absolute } \\
\text { Percentage }\end{array}$ & $\begin{array}{l}-1 \cdot 16 \\
-1 \cdot 44\end{array}$ & $\begin{array}{l}3.98 \\
5.37\end{array}$ \\
\hline
\end{tabular}

Coll, collected flow; D, Doppler; EM, electromagnetic cuff.

that obtained by thermodilution were plotted according to the Bland and Altman method (fig 7 ). The mean and standard deviation of differences between methods (thermodilution Doppler) was $0.35(0.67) \mathrm{l} / \mathrm{min}$. There was a small but significant underestimation by the Doppler method compared with the thermodilution method $(5 \cdot 1(1 \cdot 4) 1 /$ min compared with $5.5(1.2) 1 / \mathrm{min}, \mathrm{p}<0.01)$.

\section{Discussion}

These findings show the accuracy of pulsed wave Doppler estimates of the most commonly used velocity variables (peak velocity, stroke distance, and acceleration) against electromagnetic cuff and catheter measurements in both an artificial flow rig and in the human aorta. The Doppler method we used with a new high resolution spectral analysis system gave an accurate independent measure of blood velocity. The accuracy was maintained for peak velocity and the velocity integral (stroke

Figure 4 Flow profile from the flow rig experiment showing the same beat measured by electromagnetic cuff (top) and by Doppler (bottom). The electromagnetic signal is displayed as a single line (average flow) output whereas the Doppler measurement is the spectral display of the Doppler frequency shift (proportional to velocity). The figure shows the similarity in the shape of the same beat estimated by the two techniques. Comparability of actual measurements is described in the text. distance), but there was much more variation between methods for acceleration.

The Doppler method gave accurate estimates of volume flow in an artificial flow rig compared with the absolute flow measurement when the cross sectional area of the flow rig tubing was known. In humans volume flow was compared with thermodilution estimates (through a pulmonary artery catheter), which themselves are subject to a fair degree of variability and are sources of inaccuracy. ${ }^{16}$ Despite this proviso the volume flow estimates obtained by the Doppler method compared reasonably well with the thermodilution method in the subjects we studied, and these results accord with reports of similar comparisons of thermodilution and continuous
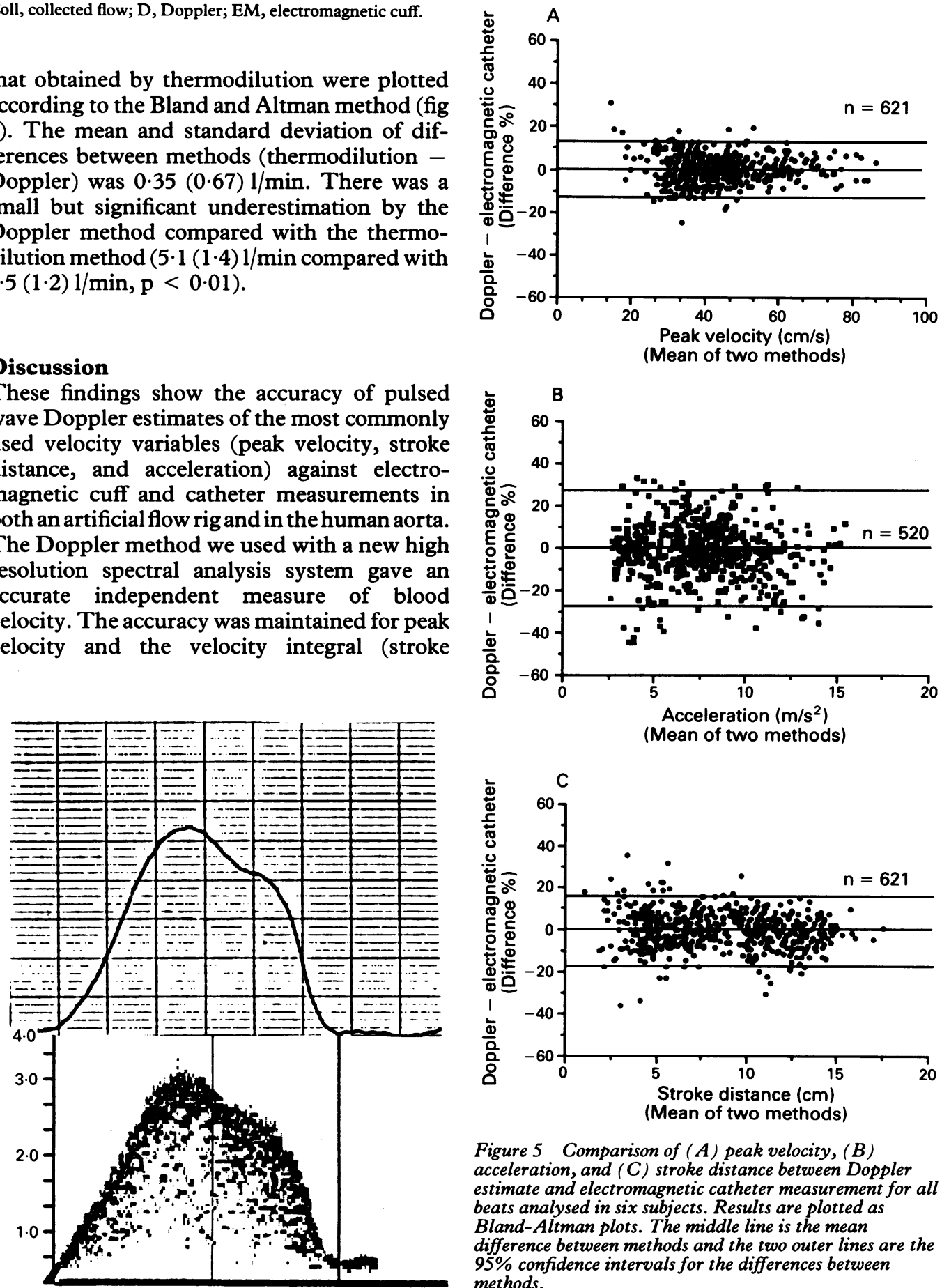

Figure 5 Comparison of $(A)$ peak velocity, $(B)$ acceleration, and $(C)$ stroke distance between Doppler estimate and electromagnetic catheter measurement for all beats analysed in six subjects. Results are plotted as Bland-Altman plots. The middle line is the mean difference between methods and the two outer lines are the 95\% confidence intervals for the differences between methods. 
Figure 6 Comparison between minute distance for all 10 beat averages recorded by Doppler and by electromagnetic catheter in six subjects. See legend to fig 5 for explanation.

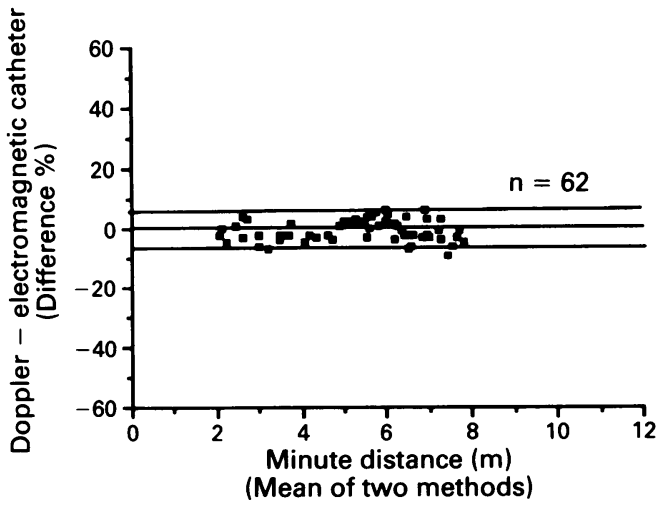

wave $^{17-19}$ and pulsed wave Doppler. ${ }^{132021}$ These and more recent reports ${ }^{102223}$ give standard deviations of differences compared with simultaneous thermodilution estimates of cardiac output in the range $8-22 \%$.

Inaccuracies in estimating volume blood flow owe more to inaccuracies in the measurement of aortic cross sectional area than to the evaluation of blood velocity itself. This was shown by a greater similarity in our results between electromagnetic and Doppler velocity variables in man and the flow rig than was seen between either of the velocity based methods and thermodilution. The small $(<10 \%$, but significant) underestimation by Doppler compared with thermodilution in both stroke volume and cardiac output is probably caused by errors in measurement of the cross sectional area of the ascending aorta.

There is no ideal reference standard for human volume blood flow measurements, but it seems that the Doppler method is a good technique in subjects without turbulent blood flow in the ascending aorta. If we assume that the cross sectional area of the ascending aorta does not change appreciably in the short to medium term or with drugs or moderate blood pressure changes ${ }^{24}$ then the ability of the Doppler method to quantify proportional changes in cardiac output will be even greater than its ability to give a single measure of cardiac output.

The advantages of high resolution spectral analysis can be seen by the greater temporal resolution shown when a pure frequency signal was rapidly changed. Spectral analysers with larger time bins, and hence lower temporal resolution, artificially blur rapidly changing blood velocities, and hence no description of an instantaneous maximal acceleration is obtainable. Our results support the approach of averaging a period of the acceleration phase

Table 5 Correlation coefficients between electromagnetic catheter and Doppler estimates for individual subjects.

\begin{tabular}{llll}
\hline Subject & Peak velocity & Acceleration & Stroke distance \\
\hline 1 & 0.96 & 0.84 & 0.97 \\
2 & 0.92 & 0.83 & 0.94 \\
3 & 0.98 & NA & 0.95 \\
4 & 0.90 & 0.84 & 0.80 \\
5 & 0.86 & 0.80 & 0.94 \\
6 & 0.94 & 0.90 & 0.90
\end{tabular}

NA, not available because of electrical interference on tape recording.

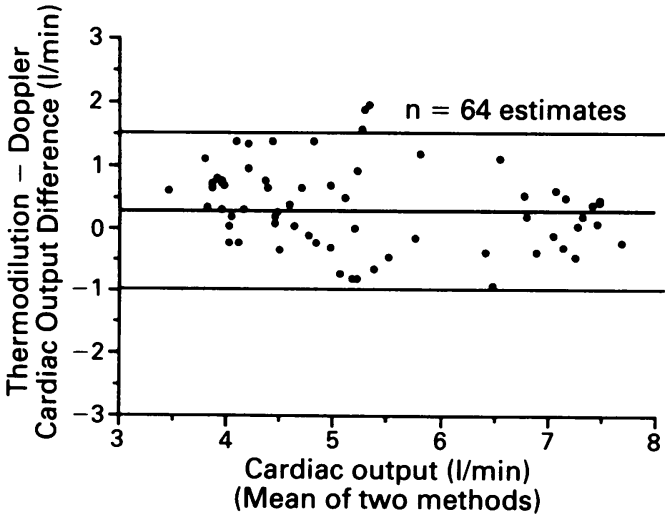

Figure 7 Bland-Altman plot for all paired measurements of cardiac output from Doppler derived measurements and simultaneous thermodilution estimates. The middle line is the mean difference between methods and the two outer lines are the $95 \%$ confidence intervals of the differences between methods.

over which an adequate number of data points can be accumulated to give a reliable estimate of acceleration. The new analyser we describe, when used in off-line high resolution mode increases the temporal resolution of Doppler signals substantially. This allows more detailed description of rapidly changing blood velocities, which would be useful in assessing acceleration, turbulence, and spatially dispersed blood velocity profiles. Most Doppler recordings in cardiological examinations are obtained on machines that combine echo and Doppler and in which the spectral analysers more commonly use a $10 \mathrm{~ms}$ time bin for FFT analysis (similar to the slow sweep setting of the Doptek analyser described here) rather than the smaller $5 \mathrm{~ms}$ time bins used in the Doptek fast sweep mode or the on-line normal resolution in our new analyser and the $<1 \mathrm{~ms}$ time bin of the high resolution mode off-line analyses. The results in table 1 show how low is the temporal resolution of these conventional analysers.

We found that acceleration was much less reliable and showed more between method variation than either stroke distance or peak velocity, and we argue that it is the least reliable of the Doppler-derived velocity measurements.

There is a need for accurate blood velocity measurements in the ascending aorta and other parts of the circulation, both to provide a relatively reliable, safe, and repeatable measure of volume flow rate, and also to investigate noninvasively blood velocity patterns themselves. Accurate non-invasive measurements of blood velocity by Doppler may be the best way of obtaining information on aortic flow patterns, blood flow turbulence, and the influence of pressure-reflected waves on ventriculoaortic coupling in heart failure and other conditions.

CM was in receipt of a Texas Instruments Computing scholarship at Green College, Oxford.

1 Hatle L, Angelsen B. Doppler ultrasound in cardiology. Philadelphia: Lea and Feibiger, 1985

2 Murphy C, Coats A, Conway J, Colditz P, Rolphe P. Doppler ultrasound signal analysis based on the TMS 320 signal processor. J Biomed Eng 1988;10:127-9.

3 Spence JD. Effects of antihypertensive agents on blood velocity: implications for atherogenesis. Can Med Assoc J 1982;127:721-4. 
4 O'Rourke M. Arterial stiffness, systolic blood pressure, and logical treatment of arterial hypertension. Hypertension logical treatment

5 Rushmer RF. Initial ventricular impulse-a potential key to cardiac evaluation. Circulation 1964;29:268-83.

6 Mehta N, Boyle G, Bennett D, et al. Hemodynamic response to treadmill exercise in normal volunteers: an assessment by Doppler ultrasonic measurement of ascending aortic blood velocity and acceleration. Am Heart J 1988; 116:1298-307.

7 Sabbah HN, Przybylski J, Albert DE, Stein PD. Peak aortic blood acceleration reflects the extent of left ventricula ischemic mass at risk. Am Heart $J$ 1987;113:885-90.

8 Levy B, Targett RC, Bardou A, McIlroy MB. Quantitative ascending aortic Doppler blood velocity in normal human subjects. Cardiovasc Res 1985;19:383-93.

9 Targett RC, Levy B, Bardou A, McIlroy MB. Simultaneous Doppler blood velocity measurements from aorta and radial artery in normal human subjects. Cardiovasc $R e s$ 1985;19:394-9.

10 Innes JA, Mills CJ, Noble MIM, et al. Validation of beat by beat pulsed Doppler measurements of ascending aortic blood velocity in man. Cardiovasc Res 1987;21:72-80.

11 Murphy C. Signal processing of Doppler ultrasound for the measurement of blood

12 Christie J, Sheldahi LM, Tristani FE, Sagar KB, Ptacin MJ, Wann S. Determination of stroke volume and cardiac output during exercise: comparison of two-dimensional and Doppler echocardiography, Fick oximetry, and thermodilution. Circulation 1987;76:539-47.

13 Ihlen H, Amlie JP, Dale J, et al. Determination of cardiac output by Doppler echocardiography. Br Heart J 1984 51:54-60.

14 Gardin JM, Tobis JM, Dabestani A, et al. Superiority of two-dimensional measurement of aortic vessel diameter in Doppler echocardiographic estimates of left ventricular stroke volume. J Am Coll Cardiol 1985;6:66-74.

15 Bland JM, Altman DG. Statistical methods for assessing agreement between two methods of clinical measurement.
Lancet 1986;i:307-10

16 Mackenzie JD, Haites NE, Rawles JM. Method of assessing the reproducibility of blood flow measurement: factors influencing the performance of thermodilution cardiac output computers. Br Heart J 1986;55:14-24.

17 Huntsman LL, Gams E, Johnson CC, Fairbanks E. Transcutaneous determination of aortic blood flow velocities in man. Am Heart J 1975;89:605-12.

18 Chandraratna PA, Nanna M, McKay C, et al. Determination of cardiac output by transcutaneous continuous-wave ultrasonic Doppler computer. Am J Cardiol 1984;53: 234-7.

19 Rose JS, Nanna M, Rahimtoola SH, Elkayam U, McKay C, Chandraratna PAN. Accuracy of determination of changes in cardiac output by transcutaneous continuouswave Doppler computer. Am J Cardiol 1984;54:1099-101.

20 Lewis JF, Kuo LC, Nelson JG, Limacher MC, Quinones MA. Pulsed Doppler echocardiographic determination of stroke volume and cardiac output: clinical validation of two new methods using the apical window. Circulation 1984;70:425-31.

21 Labovitz A Buckingham TA, Habermehl K, Nelson J, Kennedy HL, Williams GA. The effects of sampling site Kennedy HL, Williams GA. The effects of sampling site on the two-dimensional echo-Doppler determin

22 Bojanowski LMR, Timmis AD, Najm YC, Gosling RG. Pulsed Doppler ultrasound compared with thermodilution for monitoring cardiac output responses to changing left ventricular function. Cardiovasc Res 1987;21:260-8.

23 Maeda M, Yokota M, Iwase M, Miyahara T, Hayashi $\mathbf{H}$, Sotobata I. Accuracy of cardiac output measured by continuous wave Doppler echocardiography during dynamic exercise testing in the supine position in patients with coronary artery disease. J Am Coll Cardiol 1989; 13:76-83.

24 Stewart WJ, Jiang L, Mich R, Pandian N, Guerrero JL, Weyman AE. Variable effects of changes in flow rate through the aortic, pulmonary and mitral valves on valve area and flow velocity: impact on quantitative Doppler flow calculations. J Am Coll Cardiol 1985;6:653-62. 